Review Article

\title{
A Review on Bioactivities of Perilla: Progress in Research on the Functions of Perilla as Medicine and Food
}

\author{
Miho Igarashi and Yoshifumi Miyazaki \\ Center for Environment, Health and Field Sciences, Chiba University, Kashiwanoha 6-2-1, Kashiwa, Chiba 277-0882, Japan \\ Correspondence should be addressed to Yoshifumi Miyazaki; ymiyazaki@faculty.chiba-u.jp
}

Received 12 April 2013; Accepted 24 September 2013

Academic Editor: Wagner Vilegas

Copyright (C) 2013 M. Igarashi and Y. Miyazaki. This is an open access article distributed under the Creative Commons Attribution License, which permits unrestricted use, distribution, and reproduction in any medium, provided the original work is properly cited.

Perilla is a useful pharmaceutical and food product and is empirically consumed by humans. However, its properties have not been evaluated extensively. In this review, we summarize the progress made in research, focusing on the bioactivities of perilla. There are many in vitro and animal studies on the cytostatic activity and antiallergic effects, respectively, of perilla and its constituents. However, its influence on humans remains unclear. Hence, investigating and clarifying the physiological effects of perilla and its constituents on humans are imperative in the future to adhere to the ideals of evidence-based medicine.

\section{Introduction}

Perilla is a type of aromatic vegetables. Asian people preferred consuming it since ancient times. It was used to treat fish and crab poisoning symptoms according to Chinese classics [1]. This information passed on from China throughout Asia; thereafter, perilla is used as a traditional medicine and functional food in Asia.

In traditional medicine, aromatic substances are often used to treat mental stress. Perilla is included as one of them, and it is also used in combination with other aromatic oriental medicines that are referred to as Kampo medicines (hereafter "Kampo"). Hangekobokuto (Chinese name: Banxia-HoupoTang), Kososan (Chinese name: Xiang-Su-San), and SuyuJiaonang (SYJN) are representative Kampo, and these are used to treat depression-related diseases and asthma [2].

It is reported that Hangekobokuto can ameliorate sleep choking syndrome [3], swallowing reflex $[4,5]$, and panic disorder [6] in humans and has antidepressant effects in mice [7]. Ito et al. [8] reported that Kososan exhibited antidepressant-like effects in murine forced swimming model, and Suyu-Jiaonang (SYJN) demonstrated antistress effects in rats [9]. However, the importance and role of perilla in these Kampo medicines remain to be clarified.

In Japan, these Kampo have been used as medicine for a long time and are now approved and used as general medicines. In recent years, Kampo has attracted attention as an alternative medicine among the foreign countries, including those in Europe and the United States, and the World Health Organization (WHO) announced that they will add a chapter on traditional medicines, including Japanese Kampo, in the eleventh edition of the International Classification of Diseases (ICD-11) in 2015. Accordingly, scientific investigations of the effects of Kampo are advancing [49]. Nonetheless, for many years, Kampo has been used in traditional Asian medicines to treat physical conditions such as stress and asthma.

We review the use of bioactive compounds from perilla varieties as medicines and foods to prevent and ameliorate illnesses.

\section{Methodology}

All published reports on physiological functions of perilla were extracted from the PubMed database.

\section{Perilla as Food and KAMPO}

Two types of perilla, (Perilla frutescens (L.) Britton var. crispa (Thunb.) H. Deane f. purpurea (Makino) Makino and Perilla frutescens (L.) Britton var. crispa (Thunb.) H. Deane viridis 
TABLE 1: Types of a perilla used for food and medicinal purposes $[10,11]$.

\begin{tabular}{ll}
\hline & Scientific name \\
\hline & Perilla frutescens (L.) Britton crispa (Thunb.) \\
Food & H. Deane f. purpurea (Makino) Makino \\
& $\begin{array}{l}\text { Perilla frutescens (L.) Britton crispa (Thunb.) } \\
\text { "Viridis crispa" } \\
\text { Perilla frutescens (L.) frutescens }\end{array}$ \\
\hline Medicine & Perilla frutescens Britton var. acuta Kudo \\
Perillae Herba & Perilla frutescens Britton crispa var. Decaisne \\
\hline
\end{tabular}

TABLE 2: The main constituents in perillae herba [10].

\begin{tabular}{ll}
\hline & Compounds \\
\hline Essential oil & $\begin{array}{l}(-) \text {-perillaldehyde, }(+) \text {-perillalcohol, } \\
(+) \text {-limonene, alpha-pinene, and trans-shisool }\end{array}$ \\
\hline Pigment & Cyanin and shisonin \\
\hline $\begin{array}{l}\text { Other } \\
\text { compounds }\end{array}$ & Rosmarinic acid, adenine, and arginine \\
\hline
\end{tabular}

crispa), are commonly added to food in Japan. In addition, perilla oil is extracted from Perilla ocymoides (Perilla frutescens (L.) Britton var. frutescens) for consumption in Asia [11] (Table 1).

In Japanese pharmacy, leaves and branches of Perilla frutescence Britt. var. acuta Kudo and Perilla frutescence Britt. var. crispa Decaisne as Perillae Herba [10] (Table 1) are defined as medicines. Table 2 shows the main constituents of perillae herba [10].

\section{Bioactivity of Perilla}

4.1. Effects of the Kampo Perillae Herba. Perillae herba has been used as an oriental medicine for many years in Asia and has been passed on through generations by experience. In Japan, evident effects of Kampo containing perillae herba have been recently reported. However, the effects of the perillae herba itself are not stated clearly. Perillae herba is included in Japanese Kampo medicines such as Hangekobokuto (Chinese name: Banxia Houpu; [36, 12-15]), Kososan (Chinese name: Xiang-Su-San; [8]), and Saibokuto (Chinese name: Chai-Pu-Tang) for the treatment of cough and stress symptoms [2]. Perillae herba is also found in Chinese Suyu-Jiaonang (SYJN), which is also used to treat mental conditions $[9,16]$.

4.2. Effects of Perilla Decoctions. Antiallergic effects of perilla decoctions have been demonstrated on mice. In these studies, perilla decoctions partly controlled IgA nephropathy [17] and type I allergies [18]. It is thought that these effects are caused by rosmarinic acid. In addition, perilla decoctions demonstrated suppressive effects on mesangioproliferative glomerulonephritis in rats [18].

In HIGA mouse model (IgA renal damage), perilla decoctions alleviated IgA nephropathy through adjustments of the mucous membrane. Moreover, rosmarinic acid, present in high quantities in perilla, was found to be a constituent of perilla decoctions, and it is believed that the maximum effect of perilla decoction is caused by rosmarinic acid [17].

The effects of rabbit anti-rat thymocyte serum were examined in a $\mathrm{BALB} / \mathrm{c}$ mouse model of mesangioproliferative glomerulonephritis, and suppressive effects were observed [19].

The positive effects of perilla decoctions were observed in ddY mice with type I allergies [18]. After the oral administration of $500 \mathrm{mg} / \mathrm{kg}$ perilla decoction and the relative amount of rosmarinic acid in a mouse model with ear passive cutaneous anaphylaxis (PCA), allergic reactions were inhibited. Because the inhibition rates of perilla decoctions and rosmarinic acid were approximately equal, it was believed that the effect of perilla decoction depended on rosmarinic acid $[18,20]$. However, comparisons of the effects of rosmarinic acid with perilla decoctions indicate additional therapeutic constituents in perilla.

4.3. Effects of Perilla Extract. In cultured murine vascular smooth muscle cells, perilla extract has been shown to induce NO production, indicating that perilla may be useful for the prevention of vascular diseases such as arteriosclerosis [21]. In addition, perilla extract increased restraint and induced cell death in human hepatoma HepG2 cells [22]. Moreover, flow cytometry and DNA microarray experiments revealed significant apoptosis and time-dependent regulation of apoptotic genes, respectively, in cells treated with perilla leaf extracts.

However, in this study the experiment was conducted in vitro with a high dose of the perilla extract; therefore, it is unclear if perilla extract is effective in vivo.

4.4. Effects of Perilla Oil. Perilla oil contains large quantities of alpha-linolenic acid, an essential fatty acid, which decreases the risk of cardiovascular diseases [23]. Several in vitro, animal, and human nutritional studies have focused on the effects of perilla oil and have compared it with those of other oils. Perilla oil increases glucose-6-phosphatase activity, improves membrane stability [24], lowers plasma triacylglycerol levels [25], and controls liver fatty acid composition [26].

Perilla oil, which is rich in $n-3$ polyunsaturated fatty acids, regulates brown and white adipose tissue metabolism in a manner different from that of safflower and fish oils and contributes to physiological activities that prevent body fat accumulation, regulate glucose metabolism in rats [27], and control serum lipid concentrations [28]. Perilla oil rich in alpha-linolenic acid is effectively desaturated and elongated to form eicosapentaenoic acid (EPA) and docosahexaenoic acid (DHA, [29]). In addition, in light- and dark-discrimination learning tests, perilla oil-administrated senescence-accelerated mice (SAMP8) group exhibited higher discriminability than safflower oil-administrated group [30]. Moreover, serum lipids in the perilla oiladministrated SAMP8 group had a significantly greater ratio of apolipoprotein A-I (ApoA-I) to ApoA-II than those in the safflower-oil administered group [31]. Perilla oil was not 
as effective as soybean oil in preventing minor recurrent aphthous stomatitis; however, in vivo, in vitro, and lifestyle studies report positive effects of perilla oil on minor recurrent aphthous stomatitis [32].

\section{Effects of Perilla Constituents}

Perilla contains several essential oils, including (-)perillaldehyde, (-)-perillylalcohol, (+)-limonene, alphapinene, and trans-shisool. Moreover, perilla contains the purple pigments shisonin and cyanin. Other constituents of perilla include rosmarinic acid, adenine, and arginine [10].

5.1. Effects of Perillaldehyde. In contrast with the Kampo cinnamaldehyde, perillaldehyde elicited antidepressant-like effects on the olfactory nervous system in mice that were subjected to chronic weak stress and a forced swimming test [33]. Antidepressant-like effects were evaluated by measuring the duration of immobility in the forced swimming test. Decreased duration of immobility was observed after 9 days of perillaldehyde inhalation. In contrast, the odorant cinnamaldehyde from cinnamon bark failed to decrease the duration of immobility. This antidepressant effect of perillaldehyde was abolished in mice induced with anosmia by zinc sulfate, confirming the olfactory mode of action.

In Wistar rat aortas, vasodilatation following inhibition of $\mathrm{Ca}$ channels was ameliorated by perillaldehyde, with dose-dependent aorta extensibility, and relaxation with 0.01$1 \mathrm{mM}$ treatments with prostaglandin F $2 \alpha$ or norepinephrine [34]. In addition, these effects were unchanged by treatment with NG-nitro-L-arginine methyl ester or removal of aorta endothelium. Therefore, it was suggested that perillaldehyde directly affects vascular smooth muscle. Furthermore, vasodilatation was not inhibited by the $\beta$-adrenergic-receptor blocker propranolol, the phosphodiesterase inhibitor theophylline, the delayed rectifier $\mathrm{K}^{+}$channel blocker tetraethylammonium chloride, or the ATP-sensitive $\mathrm{K}^{+}$channel blocker glibenclamide. In contrast, perillaldehyde caused vasodilatation of contracted aortas via a mechanism involving $\mathrm{Ca}^{2+}$ transport out of the cell. Perillaldehyde caused slight vasodilatation of $\mathrm{Ca}^{2+}$-ionophore (A23187) contracted aortas but inhibited highly concentrated $\mathrm{K}^{+}$-mediated vasodilatation. Given the predominance of voltage-dependent $\mathrm{Ca}^{2+}$ efflux, it was suggested that perillaldehyde acted as a $\mathrm{Ca}^{2+}$ channel inhibitor for vasodilatation.

In cultured human liver microsomes, perillaldehyde slightly inhibited the hydroxylation of bupropion (antidepressant) by the enzyme CYP2B6 [35] and inhibited Candida albicans [36]. In addition, in 293 TRPA1-HEK cells, perillaldehyde activated the transient potential A1 receptor [37], suggesting that this discovery will enable future analysis of taste properties.

Perillaldehyde inhibited the proliferation of human squamous cell carcinoma of the tongue (BroTo) and human lung adenocarcinoma (A549) [38] and demonstrated anticancer activity in PC12 cells (rat pheochromocytoma cell line [39]). Moreover, Masutani et al. [40] reported the activation of the Nrf2-Keap1 system by perillaldehyde. Furthermore, antibacterial actions (using air washers) against floating microbes were reported [41].

Perillaldehyde was found to preserve fruits and promote the antioxidant activity of blueberries [42] and Chinese bayberries [43]. Hence, these natural products may be helpful in the maintenance of crop quality and security. However, further research is required to examine the influence of perillaldehyde on flavour, texture, and harvest parameters of crops.

5.2. Perillyl Alcohol. Perillyl alcohol is the subject of only few in vitro studies. In these studies, perillyl alcohol induced cell cycle arrest and cell death in BroTo; [38], and A549 [38]. Perillyl alcohol also had inhibitory effects on HCT116 cells (human colon cancer cell line; [44]) and caused dosedependent inhibition of mammary tumor cell proliferation.

5.3. Perillic Acid. Perillic acid has been shown to inhibit proliferation of HCT116 cells (human colon cancer, [44]), mammary tumor cells [45], and PC12 cells (rat pheochromocytoma; [39]), indicating anticancer properties.

5.4. Rosmarinic Acid. Rosmarinic acid is a constituent of rosemary; therefore, there are several reports on this constituent of perilla.

It was reported that rosmarinic acid inhibits seasonal allergic rhinoconjunctivitis in humans [46]. This doubleblind study indicated that oral supplementation with rosmarinic acid is an effective intervention for patients in the age group of 21-53 years with mild seasonal allergic rhinoconjunctivitis. In this 21-day experiment, rosmarinic acid significantly increased responder rates for itchy nose, watery eyes, itchy eyes, and total symptoms and decreased neutrophil and eosinophil numbers in nasal lavage fluid. The authors suggest that rosmarinic acid may reduce treatment costs for allergic diseases.

The absorption, metabolism, and urinary excretion of rosmarinic acid after a single dose of perilla extract were determined in six healthy men (mean age $37.2 \pm 6.2$ years and mean body mass index $22.0 \pm 1.9 \mathrm{~kg} / \mathrm{m}^{2}$; [47]). In this study, rosmarinic acid was absorbed, conjugated, and methylated following intake, and a small proportion of rosmarinic acid was degraded into various constituents, including conjugated forms of caffeic acid, ferulic acid, and m-coumaric acid, which were rapidly excreted in urine.

Several studies have examined the effects of rosmarinic acid on allergic reactions in mice. In nasal mucosa of ovalbumin-sensitized BALB/c mice, rosmarinic acid and 30\% ethanol extract powder of perilla reduced the number of ear and eye rubs, the levels of IgE and histamine in the serum, inhibited protein and mRNA expression of interleukins (IL)- $1 \beta$, IL- 6 , and tumor necrosis factor- $\alpha$, and inhibited cyclooxygenase- 2 protein expression and caspase- 1 activity [48]. Moreover, rosmarinic acid blunted nuclear factor-kappa $\mathrm{B}(\mathrm{NF}-\kappa \mathrm{B}) / \mathrm{Rel} \mathrm{A}$ and caspase-1 activation. These results suggested that both rosmarinic acid and 30\% ethanol extract powder of perilla alleviate allergic inflammatory reactions such as allergic rhinitis and allergic rhinoconjunctivitis. 
TABLE 3: The main effects of perilla.

\begin{tabular}{|c|c|c|c|}
\hline Product studied & Main effects & Type of study & $\begin{array}{l}\text { Number of } \\
\text { papers }\end{array}$ \\
\hline Perilla decoction & $\begin{array}{l}\text { Type I antiallergic effect } \\
\text { Mouse ear passive cutaneous anaphylaxis (PCA) reaction } \\
\text { IgA nephropathy in HIGA mice } \\
\text { Decreased proliferation of mesangial cells }\end{array}$ & Animal & 5 \\
\hline Perilla extract & $\begin{array}{l}\text { Inhibits growth and induces NO production in vascular smooth muscle } \\
\text { cells }\end{array}$ & In vitro & 2 \\
\hline \multirow{3}{*}{ Perilla oil } & Protective effects against recurrent aphthous stomatitis $(-)$ & Human & 1 \\
\hline & Fatty acid synthesis, improved learning in the Sidman active avoidance task & Animal & 8 \\
\hline & Cytostatic activity & In vitro & 1 \\
\hline \multirow{2}{*}{ Perillaldehyde } & $\begin{array}{l}\text { Antidepressant-like effect through regulation of the olfactory nervous } \\
\text { system }\end{array}$ & Animal & 2 \\
\hline & $\begin{array}{l}\text { Vasodilatory effect } \\
\text { Cytostatic activity and so on }\end{array}$ & In vitro & 6 \\
\hline Perillyl alcohol & Cytostatic activity & In vitro & 2 \\
\hline Perillic acid & Cytostatic activity & In vitro & 2 \\
\hline \multirow{3}{*}{ Rosmarinic acid } & $\begin{array}{l}\text { Seasonal allergic rhinoconjunctivitis inhibition, absorption, metabolism, } \\
\text { degradation, and urinary excretion }\end{array}$ & Human & 2 \\
\hline & $\begin{array}{l}\text { Antiallergic effects against type I allergies } \\
\text { Antidepressant-like effect } \\
\text { Anticarcinogenic effect }\end{array}$ & Animal & 8 \\
\hline & Cytostatic activity & In vitro & 4 \\
\hline \multirow{2}{*}{ Caffeic acid } & Absorption, metabolism, degradation, and urinary excretion & Human & 1 \\
\hline & Antidepressant-like effect & Animal & 1 \\
\hline
\end{tabular}

Consistent with this report, rosmarinic acid ameliorated type I allergies in ddY mice [49] and inhibited ear-passive cutaneous anaphylaxis (PCA) reactions more effectively than the modern antiallergic drug tranilast.

In HIGA mice that spontaneously develop high levels of serum immunoglobulin A ( $\operatorname{IgA}$ ) and mesangial IgA deposition, rosmarinic acid suppressed serum IgA levels and may suppress IgA nephropathy [18].

In forced swimming tests, rosmarinic acid produced an antidepressant-like effect, at least in part via the proliferation of new-born cells in the dentate gyrus of the hippocampus [50]. Rosmarinic acid also reduced the defensive freezing behaviour of mice exposed to conditioned fear stress [51]. The authors suggest that investigating the mechanisms of these effects may help to explain the pathophysiology of such affective disorders, which would facilitate new therapeutic strategies and future development of novel anxiolytic and/or antidepressive drugs.

In other studies, rosmarinic acid demonstrated anticarcinogenic effects in a murine two-stage skin model [52]. This paper concluded that rosmarinic acid contributes to the anticarcinogenic effects of perilla via two independent mechanisms: by inhibiting inflammatory responses and potent superoxide scavenging activity. Furthermore, rosmarinic acid ameliorated LPS-induced liver injury in D-GalN-sensitized mice [53].

In vitro, rosmarinic acid inhibited mesangial-cell proliferation [54] and adriamycin-induced apoptosis in H9c2 cardiac muscle cells [55]. Moreover, Kim et al. suggested that rosmarinic acid should be viewed as a potential chemotherapeutic that inhibits cardiotoxicity in adriamycin-exposed patients. Indeed, rosmarinic acid significantly prevented 6OHDA-induced cell viability reduction [56], indicating a potential as a chemotherapeutic agent for the treatment of Parkinson's disease. Finally, rosmarinic acid inhibited the formation of reactive oxygen and nitrogen species in RAW264.7 macrophages [44].

\section{Other Constituents}

Perillaketone has been shown to activate cloned TRPA1 channels in vitro [37]. Bassoli et al. [37] suggested that these data may explain the taste properties of the plant at the molecular level and identify it as an interesting target for its culinary and pharmaceutical applications.

Carvane was shown to inhibit the transformation of Candida albicans [36] and also inhibited type I allergies in ddY mice [18].

Luteolin inhibits inflammation and allergic responses in vivo and in vitro [57], and the mechanisms of relaxant action were elucidated in isolated guinea pig tracheas [58].

The perilla constituent 1,2-Di-O-alpha-linolenoyl-snglycerol, which was extracted from the local variety, kidachirimen shiso, inhibited superoxide generation [59]. 


\section{Discussion}

In this review, we were not able to find investigations on dietary perilla, though numerous studies revealed bioactive constituents of perilla.

We searched the literature from medicine, pharmacology, and nutritional sciences to clarify the functions of perilla. We found very few studies of perilla on humans but many in vivo and in vitro investigations of its biological properties. Whereas dietary effects of perilla remain unknown, perilla oil is considered a high quality oil in nutritional sciences, which are concerned with lifestyle-related diseases.

According to numerous in vivo and in vitro studies, perilla and its constituents have anticancer, antiallergy, and antidepressant properties, and the biological activities of perilla and its constituents are increasingly well characterised (Table 3 ). Although there are several in vitro studies demonstrating anticancer properties, studies conducted in vivo are very few. Hence, further research is required to examine the effects of using perilla as a food and medicine in daily life.

Among active constituents of perilla, perillaldehyde and rosmarinic acid have received the most attention, suggesting that these compounds are important constituents. Perillaldehyde contributes to aroma and accounts for approximately $50 \%$ of the oil refined from perillas. Therefore, it is considered important to examine the effects of this compound on stress; as for Kampo and food, its pleasant aroma is well known. However, only one paper considers bioactivity from aroma alone [31]. As rosmarinic acid is a constituent of both perilla and rosemary, scientific data is available for rosmarinic acid than for the other perilla compounds. Consequently, its effects on allergies are well characterised in humans [46].

In this review, we have summarized the current progress in perilla research. Although we found abundant in vitro and animal studies, we could not find a human study regarding the traditional theory on the effects of Kampo aroma on stress and depression. Investigating and clarifying the physiological effects of perilla and its constituents on humans are imperative in the future to adhere to the ideals of evidence-based medicine (EBM).

\section{Conclusion}

Animal and in vitro studies abundantly demonstrate the effects of perilla and its constituents; however, their influence on humans is nearly unknown. In contrast, perilla has already been used as a form of oriental medicine and as food. Investigating and clarifying the physiological effects of perilla and its constituents on humans are imperative in the future to adhere to the ideals of EBM.

\section{Conflict of Interests}

The authors declare no conflict of interests.

\section{References}

[1] Y. Takahashi, Handbook of Modern Chinese Medicine II, Yakkyoku Shimbun, Tokyo, Japan, 1969, [Japanese].
[2] "Notification of the medical devices investigation office, evaluation and licensing division," in Ministry of Health LaWoJ, 2012.

[3] A. Hisanaga, T. Itoh, Y. Hasegawa et al., "A case of sleep choking syndrome improved by the Kampo extract of Hange-kobokuto," Psychiatry and Clinical Neurosciences, vol. 56, no. 3, pp. 325327, 2002.

[4] K. Iwasaki, Q. Wang, H. Seki et al., "The effects of the traditional Chinese medicine, "Banxia Houpo Tang (Hange-Koboku To)" on the swallowing reflex in Parkinson's disease," Phytomedicine, vol. 7, no. 4, pp. 259-263, 2000.

[5] K. Iwasaki, Q. Wang, T. Nakagawa, T. Suzuki, and H. Sasaki, "The Traditional Chinese Medicine Banxia Houpo Tang improves swallowing reflex," Phytomedicine, vol. 6, no. 2, pp. 103-106, 1999.

[6] N. Mantani, A. Hisanaga, T. Kogure, T. Kita, Y. Shimada, and K. Terasawa, "Four cases of panic disorder successfully treated with Kampo (Japanese herbal) medicines: kami-shoyo-san and Hange-koboku-to," Psychiatry and Clinical Neurosciences, vol. 56, no. 6, pp. 617-620, 2002.

[7] L. Luo, J. Nong Wang, L. D. Kong, Q. G. Jiang, and R. X. Tan, "Antidepressant effects of Banxia Houpu decoction, a traditional Chinese medicinal empirical formula," Journal of Ethnopharmacology, vol. 73, no. 1-2, pp. 277-281, 2000.

[8] N. Ito, T. Nagai, T. Yabe, S. Nunome, T. Hanawa, and H. Yamada, "Antidepressant-like activity of a Kampo (Japanese herbal) medicine, Koso-san (Xiang-Su-San), and its mode of action via the hypothalamic-pituitary-adrenal axis," Phytomedicine, vol. 13, no. 9-10, pp. 658-667, 2006.

[9] Q.-Q. Mao, Z. Huang, X.-M. Zhong et al., "Effects of SYJN, a Chinese herbal formula, on chronic unpredictable stressinduced changes in behavior and brain BDNF in rats," Journal of Ethnopharmacology, vol. 128, no. 2, pp. 336-341, 2010.

[10] I. Kitagawa, J. Kinjyo, H. Kuwasima et al., Pharmacognosy, Hirokawa, Tokyo, Japan, 7th edition, 2011.

[11] T. Makino, New Makino Japan Plant, Diagram Model Hokuryukan, Tokyo, Japan, 2008.

[12] A. Kaneko, S. Cho, K. Hirai et al., "Hange-koboku-to, a kampo medicine, modulates cerebral levels of 5-HT (5hydroxytryptamine), NA (noradrenaline) and DA (dopamine) in mice," Phytotherapy Research, vol. 19, no. 6, pp. 491-495, 2005.

[13] J.-M. Li, L.-D. Kong, Y.-M. Wang, C. H. K. Cheng, W.-Y. Zhang, and W.-Z. Tan, "Behavioral and biochemical studies on chronic mild stress models in rats treated with a Chinese traditional prescription Banxia-houpu decoction," Life Sciences, vol. 74, no. 1, pp. 55-73, 2003.

[14] T. Naito, H. Itoh, and M. Takeyama, "Effects of Hange-kobokuto (Banxia-houpo-tang) on neuropeptide levels in human plasma and saliva," Biological and Pharmaceutical Bulletin, vol. 26, no. 11, pp. 1609-1613, 2003.

[15] T. Naito, H. Itoh, and M. Takeyama, "Some gastrointestinal function regulatory Kampo medicines have modulatory effects on human plasma adrenocorticotropic hormone and cortisol levels with continual stress exposure," Biological and Pharmaceutical Bulletin, vol. 26, no. 1, pp. 101-104, 2003.

[16] Q.-Q. Mao, X.-M. Zhong, Z.-Y. Li, C.-R. Feng, A.-J. Pan, and Z. Huang, "Herbal formula SYJN increases neurotrophin-3 and nerve growth factor expression in brain regions of rats exposed to chronic unpredictable stress," Journal of Ethnopharmacology, vol. 131, no. 1, pp. 182-186, 2010.

[17] T. Makino, T. Ono, K. Matsuyama et al., "Suppressive effects of Perilla frutescents on IgA nephropathy in HIGA mice," 
Nephrology Dialysis Transplantation, vol. 18, no. 3, pp. 484-490, 2003.

[18] T. Maniko, T. Nakamura, T. Ono, E. Muso, and G. Honda, "Suppressive effects of Perilla frutescens on Mesangioproliferative glomerulonephritis in rats," Biological and Pharmaceutical Bulletin, vol. 24, no. 2, pp. 172-175, 2001.

[19] T. Makino, M. Ito, F. Kiuchiu, T. Ono, E. Muso, and G. Honda, "Inhibitory effect of decoction of Perilla frutescens on cultured murine mesangial cell proliferation and quantitative analysis of its active constituents," Planta Medica, vol. 67, no. 1, pp. 24-28, 2001.

[20] T. Makino, Y. Furuta, H. Fujii et al., "Effect of oral treatment of Perilla frutescens and its constituents on type-I allergy in mice," Biological and Pharmaceutical Bulletin, vol. 24, no. 10, pp. 12061209, 2001.

[21] T. Makino, T. Ono, E. Muso, and G. Honda, "Effect of Perilla frutescens on nitric oxide production and DNA synthesis in cultured murine vascular smooth muscle cells," Phytotherapy Research, vol. 16, no. 1, pp. S19-S23, 2002.

[22] C.-S. Lin, C.-L. Kuo, J.-P. Wang, J.-S. Cheng, Z.-W. Huang, and C.-F. Chen, "Growth inhibitory and apoptosis inducing effect of Perilla frutescens extract on human hepatoma HepG2 cells," Journal of Ethnopharmacology, vol. 112, no. 3, pp. 557-567, 2007.

[23] W. J. E. Bemelmans, F. A. J. Muskiet, E. J. M. Feskens et al., "Associations of alpha-linolenic acid and linoleic acid with risk factors for coronary heart disease," European Journal of Clinical Nutrition, vol. 54, no. 12, pp. 865-871, 2000.

[24] Y. Kim, S.-K. Ji, and H. Choi, "Modulation of liver microsomal monooxygenase system by dietary $n-6 / n-3$ ratios in rat hepatocarcinogenesis," Nutrition and Cancer, vol. 37, no. 1, pp. 65-72, 2000.

[25] H.-K. Kim, S. Choi, and H. Choi, "Suppression of hepatic fatty acid synthase by feeding $\alpha$-linolenic acid rich Perilla oil lowers plasma triacylglycerol level in rats," Journal of Nutritional Biochemistry, vol. 15, no. 8, pp. 485-492, 2004.

[26] H. Kim, S. Choi, H.-J. Lee, J.-H. Lee, and H. Choi, "Suppression of fatty acid synthase by dietary polyunsaturated fatty acids is mediated by fat itself, not by peroxidative mechanism," Journal of Biochemistry and Molecular Biology, vol. 36, no. 3, pp. 258264, 2003.

[27] Y. Takahashi and T. Ide, "Dietary n-3 fatty acids affect mRNA level of brown adipose tissue uncoupling protein 1, and white adipose tissue leptin and glucose transporter 4 in the rat," British Journal of Nutrition, vol. 84, no. 2, pp. 175-184, 2000.

[28] T. Ide, M. Murata, and M. Sugano, "Stimulation of the activities of hepatic fatty acid oxidation enzymes by dietary fat rich in $\alpha$ linolenic acid in rats," Journal of Lipid Research, vol. 37, no. 3, pp. 448-463, 1996.

[29] T. Ide, H. Kobayashi, L. Ashakumary et al., "Comparative effects of Perilla and fish oils on the activity and gene expression of fatty acid oxidation enzymes in rat liver," Biochimica et Biophysica Acta, vol. 1485, no. 1, pp. 23-35, 2000.

[30] M. Umezawa, A. Ohta, H. Tojo, H. Yagi, M. Hosokawa, and T. Takeda, "Dietary $\alpha$-linolenate/linoleate balance influences learning and memory in the senescence-accelerated mouse (SAM)," Brain Research, vol. 669, no. 2, pp. 225-233, 1995.

[31] M. Umezawa, T. Takeda, K. Kogishi et al., "Serum lipid concentrations and mean life span are modulated by dietary polyunsaturated fatty acids in the senescence-accelerated mouse," Journal of Nutrition, vol. 130, no. 2, pp. 221-227, 2000.

[32] K. Hamazaki, M. Itomura, T. Hamazaki, and S. Sawazaki, "Effects of cooking plant oils on recurrent aphthous stomatitis: a randomized, placebo-controlled, double-blind trial," Nutrition, vol. 22, no. 5, pp. 534-538, 2006.

[33] N. Ito, T. Nagai, T. Oikawa, H. Yamada, and T. Hanawa, "Antidepressant-like effect of l-Perillaldehyde in stress-induced depression-like model mice through regulation of the olfactory nervous system," Evidence-Based Complementary and Alternative Medicine, vol. 2011, Article ID 512697, 5 pages, 2011.

[34] S. Takagi, H. Goto, Y. Shimada et al., "Vasodilative effect of Perillaldehyde on isolated rat aorta," Phytomedicine, vol. 12, no. 5, pp. 333-337, 2005.

[35] K.-A. Seo, H. Kim, H.-Y. Ku et al., "The monoterpenoids citral and geraniol are moderate inhibitors of CYP2B6 hydroxylase activity," Chemico-Biological Interactions, vol. 174, no. 3, pp. 141146, 2008.

[36] P. McGeady, D. L. Wansley, and D. A. Logan, "Carvone and Perillaldehyde interfere with the serum-induced formation of filamentous structures in Candida albicans at substantially lower concentrations than those causing significant inhibition of growth," Journal of Natural Products, vol. 65, no. 7, pp. 953955, 2002.

[37] A. Bassoli, G. Borgonovo, S. Caimi et al., "Taste-guided identification of high potency TRPAl agonists from Perilla frutescens," Bioorganic and Medicinal Chemistry, vol. 17, no. 4, pp. 16361639, 2009.

[38] J. A. Elegbede, R. Flores, and R. C. Wang, "Perillyl alcohol and Perillaldehyde induced cell cycle arrest and cell death in BroTo and A549 cells cultured in vitro," Life Sciences, vol. 73, no. 22, pp. 2831-2840, 2003.

[39] P. J. M. Boon, D. Van der Boon, and G. J. Mulder, "Cytotoxicity and biotransformation of the anticancer drug perillyl alcohol in PC12 cells and in the rat," Toxicology and Applied Pharmacology, vol. 167, no. 1, pp. 55-62, 2000.

[40] H. Masutani, R. Otsuki, Y. Yamaguchi et al., "Fragrant unsaturated aldehydes elicit activation of the keap1/Nrf2 system leading to the upregulation of thioredoxin expression and protection against oxidative stress," Antioxidants and Redox Signaling, vol. 11, no. 5, pp. 949-962, 2009.

[41] K. Sato, S. Krist, and G. Buchbauer, "Antimicrobial effect of trans-cinnamaldehyde, (-)-Perillaldehyde, (-)-Citronellal, citral, eugenol and carvacrol on airborne microbes using an airwasher," Biological and Pharmaceutical Bulletin, vol. 29, no. 11, pp. 2292-2294, 2006.

[42] C. Y. Wang, S. Y. Wang, and C. Chen, "Increasing antioxidant activity and reducing decay of blueberries by essential oils," Journal of Agricultural and Food Chemistry, vol. 56, no. 10, pp. 3587-3592, 2008.

[43] P. Jin, X. Wu, F. Xu, X. Wang, J. Wang, and Y. Zheng, "Enhancing antioxidant capacity and reducing decay of Chinese bayberries by essential oils," Journal of Agricultural and Food Chemistry, vol. 60, no. 14, pp. 3769-3775, 2012.

[44] S. Bardon, V. Foussard, S. Fournel, and A. Loubat, "Monoterpenes inhibit proliferation of human colon cancer cells by modulating cell cycle-related protein expression," Cancer Letters, vol. 181, no. 2, pp. 187-194, 2002.

[45] S. Bardon, K. Picard, and P. Martel, "Monoterpenes inhibit cell growth, cell cycle progression, and cyclin D1 gene expression in human breast cancer cell lines," Nutrition and Cancer, vol. 32, no. 1, pp. 1-7, 1998.

[46] H. Takano, N. Osakabe, C. Sanbongi et al., "Extract of Perilla frutescens enriched for rosmarinic acid, a polyphenolic phytochemical, inhibits seasonal allergic rhinoconjunctivitis in 
humans," Experimental Biology and Medicine, vol. 229, no. 3, pp. 247-254, 2004.

[47] S. Baba, N. Osakabe, M. Natsume et al., "Absorption, metabolism, degradation and urinary excretion of rosmarinic acid after intake of Perilla frutescens extract in humans," European Journal of Nutrition, vol. 44, no. 1, pp. 1-9, 2005.

[48] H.-A. Oh, C.-S. Park, H.-J. Ahn, Y. S. Park, and H.-M. Kim, "Effect of Perilla frutescens var. acuta Kudo and rosmarinic acid on allergic inflammatory reactions," Experimental Biology and Medicine, vol. 236, no. 1, pp. 99-106, 2011.

[49] T. Makino, Y. Furuta, H. Wakushima, H. Fujii, K.-I. Saito, and Y. Kano, "Anti-allergic effect of Perilla frutescens and its active constituents," Phytotherapy Research, vol. 17, no. 3, pp. 240-243, 2003.

[50] N. Ito, T. Yabe, Y. Gamo et al., "Rosmarinic acid from Perillae herba produces an antidepressant-like effect in mice through cell proliferation in the hippocampus," Biological and Pharmaceutical Bulletin, vol. 31, no. 7, pp. 1376-1380, 2008.

[51] H. Takeda, M. Tsuji, J. Miyamoto, and T. Matsumiya, "Rosmarinic acid and caffeic acid reduce the defensive freezing behavior of mice exposed to conditioned fear stress," Psychopharmacology, vol. 164, no. 2, pp. 233-235, 2002.

[52] N. Osakabe, A. Yasuda, M. Natsume, and T. Yoshikawa, "Rosmarinic acid inhibits epidermal inflammatory responses: anticarcinogenic effect of Perilla frutescens extract in the murine two-stage skin model," Carcinogenesis, vol. 25, no. 4, pp. 549$557,2004$.

[53] N. Osakabe, A. Yasuda, M. Natsume et al., "Rosmarinic acid, a major polyphenolic component of Perilla frutescens, reduces lipopolysaccharide (LPS)-induced liver injury in Dgalactosamine (D-GalN)-sensitized mice," Free Radical Biology and Medicine, vol. 33, no. 6, pp. 798-806, 2002.

[54] T. Makino, T. Ono, E. Muso, H. Yoshida, G. Honda, and S. Sasayama, "Inhibitory effects of rosmarinic acid on the proliferation of cultured murine mesangial cells," Nephrology Dialysis Transplantation, vol. 15, no. 8, pp. 1140-1145, 2000.

[55] D.-S. Kim, H.-R. Kim, E.-R. Woo, S.-T. Hong, H.-J. Chae, and S.W. Chae, "Inhibitory effects of rosmarinic acid on adriamycininduced apoptosis in $\mathrm{H} 9 \mathrm{c} 2$ cardiac muscle cells by inhibiting reactive oxygen species and the activations of c-Jun N-terminal kinase and extracellular signal-regulated kinase," Biochemical Pharmacology, vol. 70, no. 7, pp. 1066-1078, 2005.

[56] P. Ren, H. Jiang, R. Li et al., "Rosmarinic acid inhibits 6OHDA-induced neurotoxicity by anti-oxidation in MES23.5 cells," Journal of Molecular Neuroscience, vol. 39, no. 1-2, pp. 220 225, 2009.

[57] H. Ueda, C. Yamazaki, and M. Yamazaki, "Luteolin as an anti-inflammatory and anti-allergic constituent of Perilla frutescens," Biological and Pharmaceutical Bulletin, vol. 25, no. 9, pp. 1197-1202, 2002.

[58] W.-C. Ko, C.-M. Shih, I.-J. Leu, T.-T. Chen, and J.-P. Chang, "Mechanisms of relaxant action of luteolin in isolated guinea pig trachea," Planta Medica, vol. 71, no. 5, pp. 406-411, 2005.

[59] M. Takahashi, Y. Sugiyama, K. Kawabata et al., "1,2-Di-O- $\alpha$ linolenoyl-3-O- $\beta$-galactosyl-sn-glycerol as a superoxide generation inhibitor from Perilla frutescens var. crispa," Bioscience, Biotechnology and Biochemistry, vol. 75, no. 11, pp. 2240-2242, 2011. 


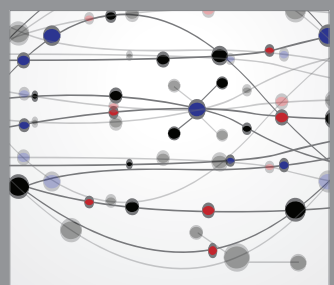

The Scientific World Journal
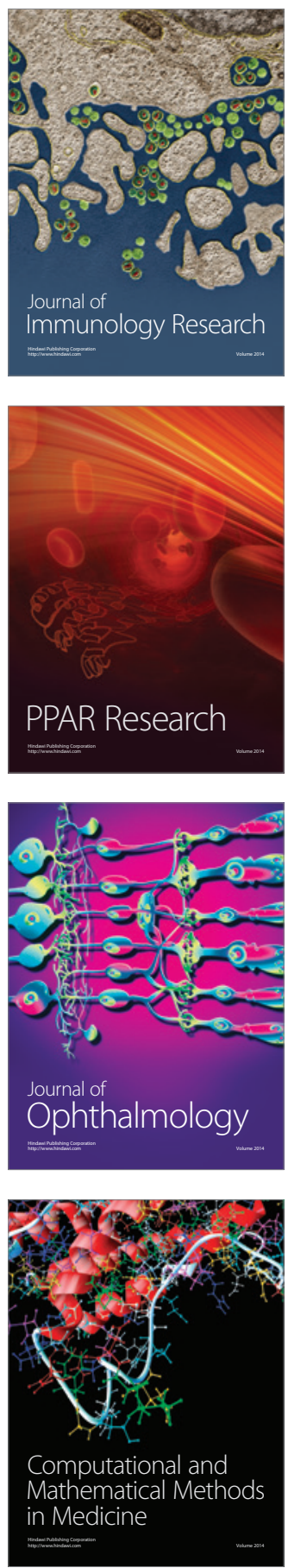

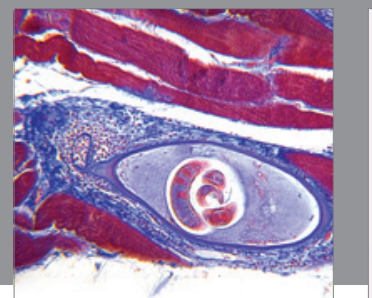

Gastroenterology

Research and Practice
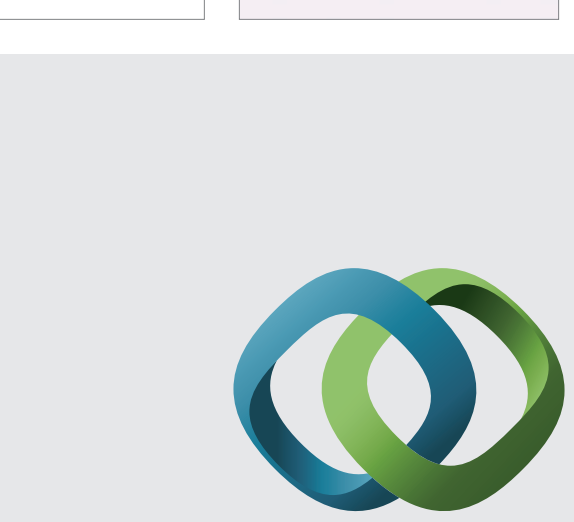

\section{Hindawi}

Submit your manuscripts at

http://www.hindawi.com
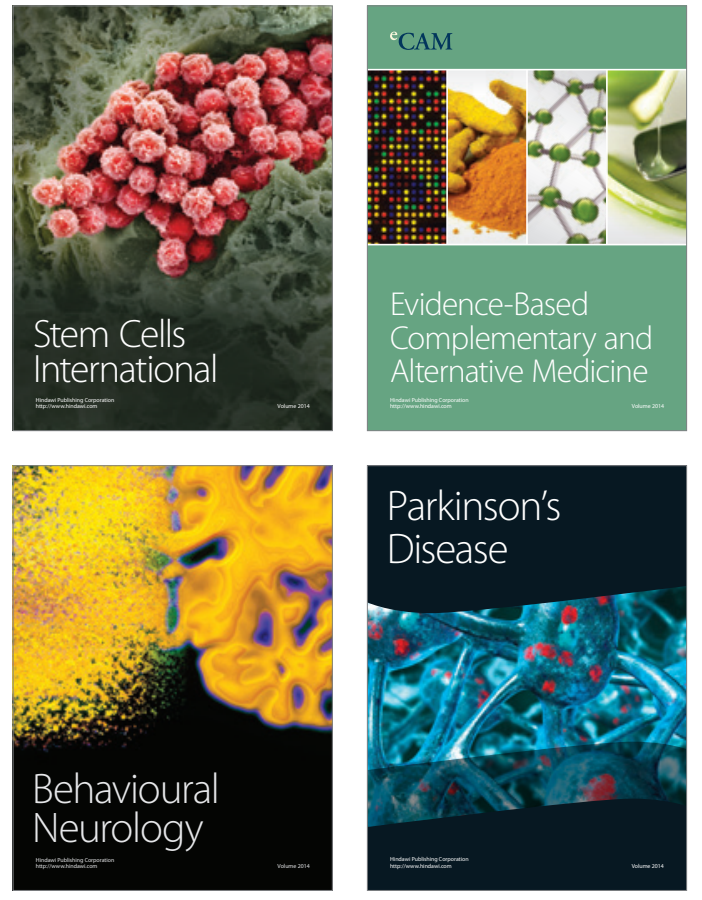
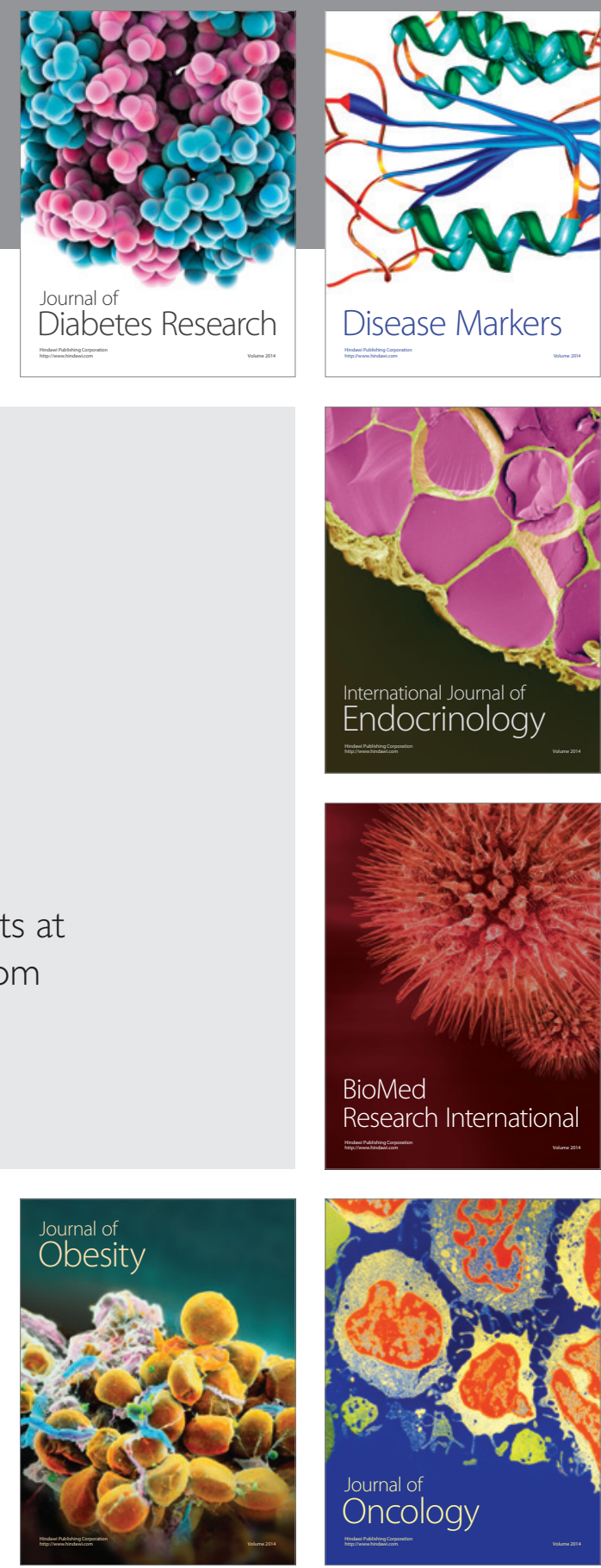

Disease Markers
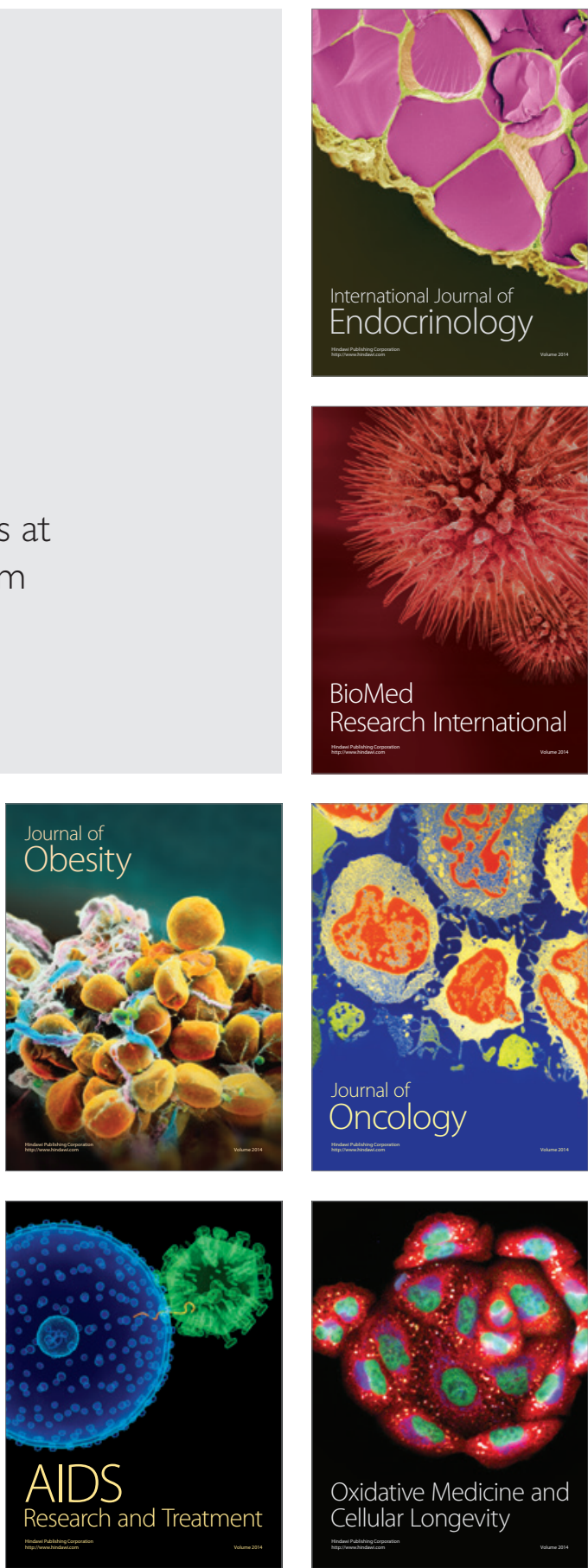